\title{
Bounds for sums of random variables when the marginal distributions and the variance of the sum are given.
}

\author{
Ka Chun Cheung \\ Department of Statistics and Actuarial Science \\ The University of Hong Kong \\ Pokfulam Road, Hong Kong \\ Phone: 852-28591987 \\ kccg@hku.hk
}

\author{
Steven Vanduffel \\ Faculty of Economics \\ Vrije Universiteit Brussel (VUB) \\ Pleinlaan 2, BE-1080 Brussels, Belgium \\ Phone: 32-497449812 \\ steven.vanduffel@vub.ac.be
}

\begin{abstract}
In this paper we establish several relations between convex order, variance order, and comonotonicity. In the first part, we extend Cheung (2008b) to show that when the marginal distributions are fixed, a sum with maximal variance is in fact a comonotonic sum. Thus the convex upper bound is achieved if and only if the marginal variables are comonotonic. Next, we study the situation where besides the marginal distributions, the variance of the sum is also fixed. Intuitively one expects that adding this information may lead to a bound that is sharper than the comonotonic upper bound. However, we show that such upper bound does not even exist. Nevertheless, we can still identify a special dependence structure known as upper comonotonicity, in which case the sum behaves like a convex largest sum in the upper tail. Finally, we investigate when the convex order is equivalent to the weaker variance order.

Throughout this paper, interpretations and significance of the results in terms of portfolio risks will be emphasized.
\end{abstract}

Key words: Copula, comonotonicity, Value-at-Risk, convex bounds, tail-end correlations, worst-case scenario

\section{Introduction}

Consider a portfolio of risks $\left(X_{1}, \ldots, X_{n}\right)$ with marginal distributions $F_{i}(i=$ $1, \ldots, n)$. We are often interested in the distribution of the sum $S=X_{1}+$ $\ldots+X_{n}$, for example when determining various risk measures of $S$ like the Value-at-Risk (VaR) and the Tail Value-at-Risk (TVaR). This requires not only the knowledge of the marginal distributions but also the dependence structure among the risks. However, the latter is often unknown, only partially known or known but difficult to work with. In this case, it is common to overcome the difficulty by identifying the least favorable dependence structure that corresponds 
to a "largest" sum $S$ (an upper bound) in the sense that all risk averse decision makers would make conservative decisions when using $S$ in these cases.

Bounds for sums of risks when only the marginal distributions are given have been studied in the literature extensively; see e.g. Denuit et al. (2005) for an overview of the most important theoretical results as well as Embrechts and Pucetti (2006) for a recent application in the context of Solvency II. Specifically, it is well-known that when the underlying risks are non-compensating (comonotonic), the sum $S$ will be the largest with respect to the convex order, and hence riskiest to all risk averse decision makers. In particular, the sum $S$ will exhibit maximal variance when the underlying risks are comonotonic. Cheung (2008b) proved that the reciprocal holds as well: if the sum $S$ is maximal with respect to convex order, then it must be a comonotonic sum, provided that each underlying risk is integrable and continuous. In the first part of this paper, we extend Cheung (2008b)'s result to show that if $S$ is a sum with maximal variance then it must be a comonotonic sum. The continuity assumption is not needed any longer, but as we make use of the variance of the sum we now need to impose square integrability on each risk . This result suggests that detecting maximal variance is meaningful when identifying the most risky situation of a portfolio.

When only the marginal distributions are given, it often holds that for the largest convex (comonotonic) sum $S$ many risk measures (such as VaR and TVaR) are additive. Hence while comonotonicity is a "prudent" dependence structure, it does not reflect the common intuition that adding risks should bring some benefit. Hence in the second part of this paper, we study the situation where besides the marginal distributions of the individual risks, the variance of the sum $S$ is also provided. We seem to be among the first to do so. Note that in practical situations it often occurs that besides the marginal distributions one also knows the variance of the sum $S$, see for example Vandendorpe et al. (2008) for a recent study when $S$ represents a sum of credit default risks. Intuitively one expects that adding the new information on variance may lead to a convex upper bound that is sharper than the comonotonic one.

Unfortunately, in this paper we show that in such a situation there does not exist a largest sum in the sense that not all risk averse decision makers will ever agree on what the most risky situation for the portfolio is. Nevertheless, we show that a so-called upper comonotonic dependence structure can still be found that ensures that $S$ behaves like a convex largest sum in the upper tail, and we relate this to an ordering concept which we label as tail convex order. Broadly speaking our results imply that while not all risk averse decision makers will consider such upper comonotonic sum $S$ as the most risky random variable, at least those risk averse decision makers who are mostly concerned with the variability in the right tail of the portfolio sum, will consider the upper comonotonic sum as the largest and riskiest sum. 
As a natural development, in the final part of this paper we study under which circumstances the variance order and the convex order will be equivalent. In such instance we can compare the riskiness of portfolios in terms of convex order by simply comparing the variance which is simpler to do. We show that in a variety of contexts the two orderings are in fact equivalent.

The rest of the paper is structured as follows. In Section 2 we summarize the results that we need in the remainder of the paper regarding the ordering of risks, comonotonicity and upper comonotonicity, and we also introduce the notion of tail convex order. Notation and conventions will be established as well. In Section 3, we analyze the equivalence of maximal variance and comonotonicity. Next in Section 4, we study the situation where besides the marginal distributions of the individual risks also the variance of the sum $S$ is provided. Finally in Section 5 we identify and discuss various relations that exist between the variance order and the convex order. In particular, we show that the two orderings are in fact equivalent in a variety of situations.

\section{Preliminaries}

Throughout this paper, all random variables are defined on a common probability space $(\Omega, \mathcal{F}, \mathbb{P})$. Given $n$ one-dimensional distribution functions $F_{1}, \ldots, F_{n}$, we denote by $\mathcal{R}\left(F_{1}, \ldots, F_{n}\right)$ the Fréchet space of all $n$-dimensional random vectors with $F_{1}, \ldots, F_{n}$ as their marginal distribution functions. Sometimes we will also use the shorthand notation $\mathcal{R}$ for denoting $\mathcal{R}\left(F_{1}, \ldots, F_{n}\right)$.

Furthermore, given any random variable $X$ or random vector $\mathbf{X}$, we use $F_{X}$ or $F_{\mathbf{X}}$ to denote its distribution function, unless otherwise stated. When we say that the $k$-th moment of a given distribution $F_{X}$ is assumed to exist we mean that the $k$-th moment of $X$ exists $(k=1,2, \ldots)$. Finally, whenever mentioned all expectations and other statistical quantities used are assumed to exist.

In this section, we recall some basic definitions and results about stochastic orders, comonotonicity, and upper comonotonicity that are needed for the rest of this paper.

\subsection{Ordering risks}

Definition 1 (Convex ordering) The random variable $X$ is said to precede another random variable $Y$ in the convex order sense, written as $X \leq_{c x} Y$, if and only if

$$
\mathbb{E}[X]=\mathbb{E}[Y] \quad \text { and } \quad \mathbb{E}\left[(X-d)_{+}\right] \leq \mathbb{E}\left[(Y-d)_{+}\right] \quad \text { for all } d \in \mathbb{R}
$$

where $(\cdot)_{+}=\max (\cdot, 0)$.

It follows from the definition that convex order represents the common preference of risk averse decision makers when choosing between risks with equal 
expectations under the Expected Utility framework of von Neumann \& Morgenstern. Hence the relationship $X \leq_{c x} Y$ can be interpreted as " $X$ is less dangerous than $Y$ ", see Denuit et al. $(1999,2005)$ or Dhaene et al. (2006) for further discussion. The intimate connection between the convex order and the comparison between risks becomes also visible when considering TVaR. Dhaene et al. (2006) have shown that

$$
X \leq_{c x} Y \Longleftrightarrow \operatorname{TVaR}_{p}[X] \leq \operatorname{TVaR}_{p}[Y] \quad \text { for all } p \in(0,1),
$$

where the Tail Value-at-Risk (TVaR) at level $p$ of a risk $Z$ is defined as

$$
\operatorname{TVaR}_{p}[Z]=\frac{1}{1-p} \int_{p}^{1} \operatorname{VaR}_{q}[Z] d q,
$$

in which $\operatorname{VaR}_{p}[Z]$ denotes the Value-at-Risk (VaR) at level $p$ of $Z$ and is equal to $\inf \left\{x \in \mathbb{R} \mid F_{Z}(x) \geq p\right\}$.

When $X$ and $Y$ are square integrable, $X \leq_{c x} Y$ implies that $\operatorname{Var}(X) \leq$ $\operatorname{Var}(Y)$. The latter is represented by the notation $X \leq_{\text {var }} Y$, and we say that $X$ is smaller than $Y$ in the variance order. Obviously, $X \leq_{\text {var }} Y$ does not imply $X \leq_{c x} Y$ in general. Nevertheless, we will demonstrate in Section 4 that they could be equivalent under some special situations.

We will also make use of a weaker ordering concept, which we label as tail convex order

Definition 2 (Tail convex ordering) The random variable $X$ is said to precede another random variable $Y$ in the tail convex order sense with index $k \in \mathbb{R}$, written as $X \leq_{t c x(k)} Y$, if the following conditions hold:

$$
\mathbb{E}[X]=\mathbb{E}[Y] \quad \text { and } \quad \mathbb{E}\left[(X-d)_{+}\right] \leq \mathbb{E}\left[(Y-d)_{+}\right] \quad \text { for all } d \geq k \in \mathbb{R} .
$$

Broadly speaking, $X \leq_{t c x(k)} Y$ can be interpreted as " $X$ is less dangerous than $Y$ beyond the threshold $k$ ". Of course we have that for all $k \in \mathbb{R}$ :

$$
X \leq_{c x} Y \Rightarrow X \leq_{t c x(k)} Y,
$$

and also that for $k_{1}<k_{2} \in \mathbb{R}$ :

$$
X \leq_{t c x\left(k_{1}\right)} Y \Rightarrow X \leq_{t c x\left(k_{2}\right)} Y,
$$

which reflects that the larger the threshold value $k$ the weaker the tail convex ordering concept is (more pairs of risks can be ordered).

\subsection{Comonotonicity}

Comonotonicity describes a very special dependence structure among several random variables in the sense that random variables are comonotonic if they always move in the same direction. More precisely, we say a subset $C$ of $\mathbb{R}^{n}$ 
is comonotonic if $\left(s_{i}-t_{i}\right)\left(s_{j}-t_{j}\right) \geq 0$ for all $i, j$ whenever $\left(s_{1}, \ldots, s_{n}\right)$ and $\left(t_{1}, \ldots, t_{n}\right)$ belong to $C$. A random vector $\left(X_{1}, \ldots, X_{n}\right)$ is called comonotonic if there is a comonotonic set $C \subseteq \mathbb{R}^{n}$ such that $\mathbb{P}\left(\left(X_{1}, \ldots, X_{n}\right) \in C\right)=1$. Equivalently, $\left(X_{1}, \ldots, X_{n}\right)$ is comonotonic if there is a random variable $Z$ and non-decreasing functions $f_{1}, \ldots, f_{n}$ such that

$$
\left(X_{1}, \ldots, X_{n}\right) \stackrel{d}{=}\left(f_{1}(Z), \ldots, f_{n}(Z)\right) .
$$

A useful characterization of comonotonicity in terms of the joint distribution function is that $\left(X_{1}, \ldots, X_{n}\right)$ is comonotonic if and only if for all real numbers $x_{1}, \ldots, x_{n}$ :

$$
F_{X_{1}, \ldots, X_{n}}\left(x_{1}, \ldots, x_{n}\right)=\min \left\{F_{X_{1}}\left(x_{1}\right), \ldots, F_{X_{n}}\left(x_{n}\right)\right\}
$$

Throughout this paper, we use the notation $\mathbf{X}^{c}=\left(X_{1}^{c}, \ldots, X_{n}^{c}\right)$ to indicate a comonotonic random vector, and the corresponding comonotonic sum $X_{1}^{c}+\cdots+$ $X_{n}^{c}$ will be denoted by $S^{c}$. An interesting property of comonotonicity is that VaR, TVaR (and by extension other distortion risk measures) are comonotonic additive:

$$
\operatorname{VaR}_{p}\left[S^{c}\right]=\sum_{i=1}^{n} \operatorname{VaR}_{p}\left[X_{i}^{c}\right] \quad \text { and } \quad \operatorname{TVaR}_{p}\left[S^{c}\right]=\sum_{i=1}^{n} \operatorname{TVaR}_{p}\left[X_{i}^{c}\right]
$$

For an overview of the basic properties of comonotonicity, we refer to Denneberg (1994), Dhaene et al. $(2002,2008)$ and Denuit et al. (2005). Some recent work about the applications of comonotonicity in finance and actuarial science can be found in Campana (2007), Deelstra et al. (2008, 2010), Vanduffel et al. (2008a,b) and Vanmaele et al (2004), amongst others.

\subsection{Upper comonotonicity}

The concept of upper comonotonicity was first studied by Cheung (2009) as a generalization of the classical concept of comonotonicity. We say that a random vector $\mathbf{X}=\left(X_{1}, \ldots, X_{n}\right)$ is upper comonotonic with threshold $\mathbf{a} \in(\mathbb{R} \cup\{-\infty\})^{n}$ if the following holds:

1. $\{\mathbf{X}(\omega) \mid \omega \in \Omega\} \cap U(\mathbf{a})$ is a comonotonic subset of $\mathbb{R}^{n}$;

2. $\mathbb{P}(\mathbf{X} \in U(\mathbf{a}))>0$;

3. $\{\mathbf{X}(\omega) \mid \omega \in \Omega\} \cap R(\mathbf{a})$ is empty.

Here, $U(\mathbf{a}):=\left(a_{1}, \infty\right) \times \cdots \times\left(a_{n}, \infty\right), R(\mathbf{a}):=\mathbb{R}^{n} \backslash(U(\mathbf{a}) \cup L(\mathbf{a}))$ where $L(\mathbf{a}):=\left(-\infty, a_{1}\right] \times \cdots \times\left(-\infty, a_{n}\right]$. Loosely speaking upper comonotonicity reflects that while in normal markets risks can be correlated with each other in a normal range, they may become extremely correlated during crisis periods. Hence upper comonotonicity reflects situations where risks move together in bad times. For example, while financial stock markets are typically presenting some 
positive correlation in normal periods, they become heavily correlated when markets are collapsing. The same holds true for a portfolio of credit risks where the collapse of a few major credit counterparts may be contagious and affect a whole portfolio leading to a sharp increase in the default statistics, see also Vandendorpe et al. (2008). We will use the notation $\mathbf{X}^{u c, \mathbf{a}}=\left(X_{1}^{u c, \mathbf{a}}, \ldots, X_{n}^{u c, \mathbf{a}}\right)$ to indicate that a random vector is upper-comonotonic with threshold a and the corresponding (upper-comonotonic) sum $X_{1}^{u c, \mathbf{a}}+\cdots+X_{n}^{u c, \mathbf{a}}$ will be denoted by $S^{u c, \mathbf{a}}$.

Upper-comonotonicity shares many nice properties of the classical notion of comonotonicity. Cheung (2008b) showed that VaR, TVaR are upper-comonotonic additive, provided that the probability level is greater than $F_{\mathbf{X}^{u c, a}}(\mathbf{a})$. The next lemma presents another useful property of comonotonic and upper-comonotonic random vectors.

Lemma 3 (Property of upper-comonotonicity) Suppose that $\left(Y_{1}, \ldots, Y_{n}\right)$ is comonotonic, and $S=Y_{1}+\cdots+Y_{n}$. For any $d \in \mathbb{R}$, there exist some constants $d_{1}, \ldots, d_{n}$ with $d_{1}+\cdots+d_{n}=d$ such that

$$
\mathbb{E}(S-d)_{+}=\sum_{i=1}^{n} \mathbb{E}\left(Y_{i}-d_{i}\right)_{+}
$$

If $\left(Y_{1}, \ldots, Y_{n}\right)$ is upper comonotonic with threshold $\left(a_{1}, \ldots, a_{n}\right)$, the above property remains valid for $d \geq a_{1}+\cdots+a_{n}$.

The first part of the lemma can be found in Kaas et al. (2002). The second part concerning upper comonotonicity is taken from Dong et al. (2010).

\section{Maximal variance and comonotonicity}

In this section, all random vectors considered belong to the same Fréchet space $\mathcal{R}\left(F_{1}, \ldots, F_{n}\right)$. The following result essentially states that within $\mathcal{R}\left(F_{1}, \ldots, F_{n}\right)$ the comonotonic sum is the least favorable from the point of view of risk averse decision makers. A proof for it can be found in e.g. Kaas et al. (2002).

Proposition 4 (Comonotonicity $\Rightarrow$ Largest convex sum) For any random vector $\left(X_{1}, \ldots, X_{n}\right) \in \mathcal{R}\left(F_{1}, \ldots, F_{n}\right)$ it holds that

$$
X_{1}+\cdots+X_{n} \leq_{c x} X_{1}^{c}+\cdots+X_{n}^{c}
$$

The proposition makes clear that for sums of random variables with given marginal distribution functions but unknown dependence structure, imposing comonotonic dependence leads to an overestimation of the risk and thus allows "safe" decision making. This property of a comonotonic sum together with its intrinsic analytical tractability (see equation (4) for instance) has been exploited in a series of papers dealing with a wide range of problems in finance and actuarial science; see Deelstra et al (2010) for a recent account. 
In this context it becomes useful to characterize comonotonicity further. Under the assumption that each marginal distribution is continuous with finite mean, Cheung (2008b) proved that the largest convex sum exhibits the comonotonic dependence structure:

Proposition 5 (Comonotonicity $\Leftrightarrow$ Largest convex sum) Assume that each $F_{i}$ is continuous with finite mean. Then $\left(X_{1}, \ldots, X_{n}\right) \in \mathcal{R}\left(F_{1}, \ldots, F_{n}\right)$ is comonotonic if and only if for every random vector $\left(X_{1}^{\prime}, \ldots, X_{n}^{\prime}\right) \in \mathcal{R}\left(F_{1}, \ldots, F_{n}\right)$ it holds that

$$
X_{1}^{\prime}+\cdots+X_{n}^{\prime} \leq_{c x} X_{1}+\cdots+X_{n}
$$

Cheung's proof of this result is rather technical. In this paper we will give a direct and simpler proof of this result without invoking the continuity assumption but under an extra assumption that each $X_{i}$ has finite variance. With this additional integrability assumption, we can further strengthen the characterization of comonotonicity by replacing the convex ordering in Proposition 5 by the weaker variance order.

Theorem 6 (Comonotonicity $\Leftrightarrow$ Largest variance) Assume that each $F_{i}$ has finite variance. Then $\left(X_{1}, \ldots, X_{n}\right) \in \mathcal{R}\left(F_{1}, \ldots, F_{n}\right)$ is comonotonic if and only if for every random vector $\left(X_{1}^{\prime}, \ldots, X_{n}^{\prime}\right) \in \mathcal{R}\left(F_{1}, \ldots, F_{n}\right)$ it holds that

$$
X_{1}^{\prime}+\cdots+X_{n}^{\prime} \leq_{\text {var }} X_{1}+\cdots+X_{n} .
$$

Proof. Step 1: Let us denote by $r\left(X_{i}, X_{j}\right)$ the Pearson's correlation coefficient of the random variables $X_{i}$ and $X_{j}$. In this step we first show that $\left(X_{i}, X_{j}\right)$ is comonotonic if and only if $r\left(X_{i}, X_{j}\right)$ is maximal. While a proof can be found in From Dhaene et al. (2002), the proof of their "if" part is not complete and requires modification. Here we provide a complete proof of it. Without losing generality, we assume that $i=1$ and $j=2$. From Dhaene et al. (2002), the maximality of $r\left(X_{1}, X_{2}\right)$ implies that

$$
\int_{-\infty}^{+\infty}\left[F_{X_{1}^{c}, X_{2}^{c}}(x, d-x)-F_{X_{1}, X_{2}}(x, d-x)\right] d x=0 \quad \text { for all } d .
$$

The integrand is non-negative by the Fréchet-Höeffding inequality. From this Dhaene et al. (2002) concluded that for all $x$ and $d$,

$$
F_{X_{1}, X_{2}}(x, d-x)=F_{X_{1}^{c}, X_{2}^{c}}(x, d-x)=\min \left\{F_{X_{1}}(x), F_{X_{2}}(d-x)\right\},
$$

and hence $F_{X_{1}, X_{2}}=F_{X_{1}^{c}, X_{2}^{c}}$. However, we can indeed only conclude from (6) that

$$
F_{X_{1}, X_{2}}(x, d-x)=\min \left\{F_{X_{1}}(x), F_{X_{2}}(d-x)\right\} \quad \text { for all } x \text { outside } N_{d},
$$

where $N_{d}$ is a subset of the real line with zero Lebesgue measure, and this null set depends on the choice of $d$. In general, the set of all $(a, b)$ such that 
$F_{X_{1}, X_{2}}(a, b) \neq \min \left\{F_{X_{1}}(a), F_{X_{2}}(b)\right\}$ may be very large. Hence in order to show that $F_{X_{1}, X_{2}}(a, b)=\min \left\{F_{X_{1}}(a), F_{X_{2}}(b)\right\}$ for all $a$ and $b$, further work is required. To this end, we make use of the right-continuity of $F_{X_{1}}$ and $F_{X_{2}}$. Fix any real numbers $a$ and $b$. Let $d=a+b$. For each positive integer $n$ we choose $a_{n} \in(a, a+1 / n) \cap N_{d+1 / n}^{c}$. This is always possible because if $(a, a+1 / n) \cap N_{d+1 / n}^{c}$ is empty, then the Lebesgue measure of $N_{d+1 / n}$ is non-zero. Next let $b_{n}=$ $d+1 / n-a_{n}$. Then $a_{n}>a, b_{n}>b$ and $a_{n} \rightarrow a, b_{n} \rightarrow b$. By passing to a subsequence if necessary, we may assume that $a_{n} \searrow a$ and $b_{n} \searrow b$. By right continuity we have

$$
\lim _{n \rightarrow \infty} F_{X_{1}, X_{2}}\left(a_{n}, b_{n}\right)=F_{X_{1}, X_{2}}(a, b)
$$

and also

$$
\lim _{n \rightarrow \infty} F_{X_{1}, X_{2}}\left(a_{n}, b_{n}\right)=\lim _{n \rightarrow \infty} \min \left\{F_{X_{1}}\left(a_{n}\right), F_{X_{2}}\left(b_{n}\right)\right\}=\min \left\{F_{X_{1}}(a), F_{X_{2}}(b)\right\} .
$$

Therefore, $F_{X_{1}, X_{2}}(a, b)=\min \left\{F_{X_{1}}(a), F_{X_{2}}(b)\right\}$ for all real numbers $a, b$, and hence $\left(X_{1}, X_{2}\right)$ is comonotonic.

Step 2: Now we are ready to complete of proof of Theorem 6. The "only if" part follows from Proposition 4. For the "if" part, suppose that $\operatorname{Var}\left(\sum_{j=1}^{n} X_{j}\right)$ is maximal but that $\left(X_{1}, \ldots, X_{n}\right)$ is not comonotonic. From Step 1 it follows that at least one $r\left(X_{i}, X_{j}\right)$ is not maximal. Hence we find that $\operatorname{Var}\left(\sum_{j=1}^{n} X_{j}\right)<$ $\operatorname{Var}\left(\sum_{j=1}^{n} X_{j}^{c}\right)$ must hold, which is a contradiction. This completes the proof.

Alternatively, Theorem 6 also follows from a result by Cuesta and Matran (1989), which asserts, in a Hilbert space setting, that $\mathbb{E}\left[\left(X_{1}-X_{2}\right)^{2}\right]$ attains its minimum value over all pairs $\left(X_{1}, X_{2}\right)$ with prescribed marginal distributions with finite second moments precisely when $\left(X_{1}, X_{2}\right)$ is comonotonic. The essence in this work is that $\mathbb{E}\left[X_{1} X_{2}\right]$ is maximized over all pairs $\left(X_{1}, X_{2}\right)$ with prescribed marginal distributions precisely when $\left(X_{1}, X_{2}\right)$ is comonotonic, which can also be regarded as a version of Hardy-Littlewood inequalities found in Föllmer and Schied (2004, Theorem A.24). The proof provided here is more transparent and elementary.

\section{Bounds when the marginal distributions and the variance of the sum are fixed}

In practice it often occurs that besides the marginal distributions, one also knows the variance of its corresponding sum $S$. Indeed while the distribution function and most risk measures of $S$ are often difficult to obtain, its variance may be readily available. Intuitively one may then expect that adding this new information may sharpen the comonotonic bound discussed above. So a natural question then is that if the variance of the sum is fixed, how can we achieve the largest convex sum and how does this relate to the comonotonic bound we discussed in the previous section. 


\subsection{General consideration}

To begin with, we fix a Fréchet space $\mathcal{R}=\mathcal{R}\left(F_{1}, \ldots, F_{n}\right)$ where it is assumed that all the $F_{i}(i=1,2, \ldots, n)$ have finite variance. Then we define a subset containing the random vectors with a fixed variance for the sum of its components. Formally

$$
\mathcal{R}_{K}=\left\{\left(X_{1}, \ldots, X_{n}\right) \in \mathcal{R} \mid \operatorname{Var}\left(X_{1}+\cdots+X_{n}\right)=K\right\},
$$

where $K$ is always taken such that

Cardinality of $\left\{\right.$ Distribution of $\left.X_{1}+\cdots+X_{n} \mid\left(X_{1}, \ldots, X_{n}\right) \in \mathcal{R}_{K}\right\}>1$.

For instance, $K$ cannot be the variance of the comonotonic sum. We aim to find some $\left(Y_{1}, \ldots, Y_{n}\right) \in \mathcal{R}_{K}$ such that

$$
X_{1}+\cdots+X_{n} \leq_{c x} Y_{1}+\cdots+Y_{n}
$$

for all $\left(X_{1}, \ldots, X_{n}\right) \in \mathcal{R}_{K}$. Surprisingly, we have the following negative result:

Theorem 7 (No convex largest sum exists in $\mathcal{R}_{K}$ ) There does not exist a maximal vector in $\mathcal{R}_{K}$ with respect to the convex order.

Proof. We prove the theorem by contradiction. Suppose that $\left(Y_{1}, \ldots, Y_{n}\right)$ is maximal in $\mathcal{R}_{K}$ with respect to the convex order. Let $S_{Y}$ be the sum $Y_{1}+\cdots+Y_{n}$. Let $\left(X_{1}, \ldots, X_{n}\right)$ be an arbitrary random vector in $\mathcal{R}_{K}$, and let $S_{X}$ be the sum $X_{1}+\cdots+X_{n}$. We claim that $S_{X} \stackrel{d}{=} S_{Y}$. To see this, we follow the idea of Denuit et al. (2005, p. 151). By the identity

$$
\frac{1}{2} \operatorname{Var}(S)=\int_{-\infty}^{\infty}\left(\mathbb{E}\left[(S-t)_{+}\right]-(\mathbb{E}[S]-t)_{+}\right) d t
$$

(cf. Kaas et al. (1994, p. 68)) and the fact that $\operatorname{Var}\left(S_{X}\right)=\operatorname{Var}\left(S_{Y}\right)=K$, we have

$$
\int_{-\infty}^{\infty}\left(\mathbb{E}\left[\left(S_{X}-t\right)_{+}\right]-\mathbb{E}\left[\left(S_{Y}-t\right)_{+}\right]\right) d t=0 .
$$

However, the integrand is positive because $S_{X} \leq_{c x} S_{Y}$. Thus $\mathbb{E}\left[\left(S_{X}-t\right)_{+}\right]=$ $\mathbb{E}\left[\left(S_{Y}-t\right)_{+}\right]$for almost all $t \in \mathbb{R}$. As both functions $t \mapsto \mathbb{E}\left[\left(S_{X}-t\right)_{+}\right]$and $t \mapsto \mathbb{E}\left[\left(S_{Y}-t\right)_{+}\right]$are decreasing, $S_{X}$ and $S_{Y}$ have the same stop-loss transforms, which implies that $S_{X} \stackrel{d}{=} S_{Y}$. We arrive at the contradiction that the sum of every random vector in $\mathcal{R}_{K}$ has the same distribution.

The theorem implies that for a portfolio of risks where the marginal distributions as well as the variance of the portfolio sum are known, it cannot occur that all risk averse decisions will ever be agreeable on what the most risky situation for the portfolio sum is.

Despite this negative result, we show in the next theorem that for a given fixed non-minimal variance, one can always find a dependence structure such 
that the corresponding sum "behaves like a comonotonic sum in the upper tail". Hence while not all risk averse decision makers will consider such upper comonotonic sum $S$ as the most risky random variable, at least those risk averse decision makers who are mostly concerned with the variability in the right tail of the portfolio sum, will consider the upper comonotonic sum as the largest and riskiest sum.

Theorem 8 (Tail convex largest sum in $\mathcal{R}_{K}$ ) If all $F_{i}$ and $F_{i}^{-1}$ are continuous, and $K$ is not minimal, then there always exists an upper comonotonic vector $\left(Y_{1}, \ldots, Y_{n}\right) \in \mathcal{R}_{K}$. Moreover there exists a threshold $k \in \mathbb{R}$ such that for all $\left(X_{1}, \ldots, X_{n}\right) \in \mathcal{R}$

$$
X_{1}+\ldots+X_{n} \leq_{t c x(k)} Y_{1}+\ldots+Y_{n}
$$

Proof. First fix an arbitrary random vector $\left(Z_{1}, \ldots, Z_{n}\right) \in \mathcal{R}$ such that $\operatorname{Var}\left(Z_{1}+\cdots+Z_{n}\right)=K$ is not minimal. The non-minimality of $K$ (for instance, $K$ is not the variance of the sum of two counter-comonotonic random vector when $n=2)$ implies the existence of some $\left(X_{1}^{\prime}, \ldots, X_{n}^{\prime}\right) \in \mathcal{R}$ such that

$$
K>l:=\operatorname{Var}\left(X_{1}^{\prime}+\cdots+X_{n}^{\prime}\right) .
$$

We also let $\left(X_{1}^{c}, \ldots, X_{n}^{c}\right) \in \mathcal{R}$ be comonotonic such that

$$
L:=\operatorname{Var}\left(X_{1}^{c}+\cdots+X_{n}^{c}\right) \geq K .
$$

If $K=L$, we may simply take $\left(X_{1}^{c}, \ldots, X_{n}^{c}\right)$ as the desired random vector. In what follows we assume that $l<K<L$. Denote the copula associated with $\left(X_{1}^{\prime}, \ldots, X_{n}^{\prime}\right)$ as $C$. Let $V_{1}, \ldots, V_{n}$ be $n$ uniform $(0,1)$ random variables with copula $C$. It is clear that $\left(F_{1}^{-1}\left(V_{1}\right), \ldots, F_{n}^{-1}\left(V_{n}\right)\right) \stackrel{d}{=}\left(X_{1}^{\prime}, \ldots, X_{n}^{\prime}\right)$. For any $\beta \in[0,1]$, define

$$
Y_{i}^{\beta}=F_{i}^{-1}\left(I_{\{U>\beta\}} U+I_{\{U \leq \beta\}} \beta V_{i}\right), \quad i=1, \ldots, n,
$$

where $U$ is any uniform $(0,1)$ random variable independent of $\left(V_{1}, \ldots, V_{n}\right), I_{\{U>\beta\}}$ and $I_{\{U \leq \beta\}}$ are indicator functions. It is easy to check that $I_{\{U>\beta\}} U+I_{\{U \leq \beta\}} \beta V_{i}$ is also uniform $(0,1)$ distributed, so $\mathbf{Y}^{\beta}:=\left(Y_{1}^{\beta}, \ldots, Y_{n}^{\beta}\right) \in \mathcal{R}$. By construction, $\mathbf{Y}^{\beta}$ is upper comonotonic with threshold $\mathbf{a}^{\beta}:=\left(F_{1}^{-1}(\beta), \ldots, F_{n}^{-1}(\beta)\right)$. Now define

$$
f(\beta):=\operatorname{Var}\left(Y_{1}^{\beta}+\cdots+Y_{n}^{\beta}\right), \quad \beta \in[0,1] .
$$

When $\beta=0,\left(Y_{1}^{0}, \ldots, Y_{n}^{0}\right)$ is comonotonic, so $f(0)=L$. When $\beta=1$, we have $\left(Y_{1}^{1}, \ldots, Y_{n}^{1}\right) \stackrel{d}{=}\left(X_{1}^{\prime}, \ldots, X_{n}^{\prime}\right)$ and hence $f(1)=l$. Moreover, $f$ is continuous by the monotone convergence theorem. Thus by the intermediate value theorem, there exists some $\beta^{*} \in(0,1)$ such that

$$
f\left(\beta^{*}\right)=\operatorname{Var}\left(Y_{1}^{\beta^{*}}+\cdots+Y_{n}^{\beta^{*}}\right)=K .
$$

Hence $\mathbf{Y}^{\beta^{*}}$ is an upper comonotonic vector in $\mathcal{R}_{K}$. 
Finally, we check that there exists some $k$ such that $\mathbf{Y}^{\beta^{*}}$ fulfills (7) for all $\left(X_{1}, \ldots, X_{n}\right) \in \mathcal{R}$. To this end, we let $k=a_{1}^{\beta^{*}}+\cdots+a_{n}^{\beta^{*}}=F_{1}^{-1}\left(\beta^{*}\right)+\cdots+$ $F_{n}^{-1}\left(\beta^{*}\right)$. Then for any $d \geq k$ and $\left(X_{1}, \ldots, X_{n}\right) \in \mathcal{R}$, Lemma 3 implies that there are some constants $d_{1}, \ldots, d_{n}$ with $d_{1}+\cdots+d_{n}=d$ such that

$$
\begin{aligned}
\mathbb{E}\left(Y_{1}^{\beta^{*}}+\cdots+Y_{n}^{\beta^{*}}-d\right)_{+} & =\sum_{i=1}^{n} \mathbb{E}\left(Y_{i}^{\beta^{*}}-d_{i}\right)_{+} \\
& =\sum_{i=1}^{n} \mathbb{E}\left(X_{i}-d_{i}\right)_{+} \\
& \geq \mathbb{E}\left(X_{1}+\cdots+X_{n}-d\right)_{+},
\end{aligned}
$$

as desired.

The copula $C$ in the above proof, which is the joint distribution of $V_{1}, \ldots, V_{n}$, is called the base copula of the upper comonotonic random vector $\left(Y_{1}^{\beta}, \ldots, Y_{n}^{\beta}\right)$. In general, different base copulas lead to different upper comonotonic random vectors in $\mathcal{R}_{K}$.

From Theorem 8 it follows that in a situation where the marginal risks present upper comonotonic behavior, the risk of the portfolio sum $S$ as measured by VaR or TVaR will be rather robust with respect to changing the correlations between the risks. More precisely, if $\left(X_{1}, \ldots, X_{n}\right)$ and $\left(X_{1}^{\prime}, \ldots, X_{n}^{\prime}\right)$ are two portfolios of upper comonotonic risks with the same marginals, the sums $S=$ $X_{1}+\cdots+X_{n}$ and $S^{\prime}=X_{1}^{\prime}+\cdots+X_{n}^{\prime}$ may have different variance but yet their VaR or TVaR will be the same whenever the probability level $p$ is larger than some threshold $p^{*}$ :

$$
\operatorname{VaR}_{p}[S]=\operatorname{VaR}_{p}\left[S^{\prime}\right]=\operatorname{VaR}_{p}\left[X_{1}\right]+\cdots+\operatorname{VaR}_{p}\left[X_{n}\right], \quad p \geq p^{*} .
$$

It also follows that, when the marginal distributions as well as the variance of the sum are known, using an upper comonotonic sum to determine capital requirements does not guarantee that diversification effects between the risks will be accounted for, i.e. when $S$ is an upper comonotonic sum we still have that $\operatorname{VaR}_{p}[S]=\sum_{i=1}^{n} \operatorname{VaR}_{p}\left[X_{i}\right]$ for all $p \geq p^{*}$ where $p^{*}$ is some non-negative threshold value.

\subsection{The two-dimensional case}

In the two-dimensional case, more can be said in relation to Theorem 8 . The Fréchet lower bound copula $C_{L}\left(u_{1}, u_{2}\right)=\max \left(0, u_{1}+u_{2}-1\right)$ corresponds to the minimal variance of the sum for all random vectors in a given Fréchet space. Hence if we replace in the proof of Theorem 8 the copula $C$ by $C_{L}$ we obtain a new function $\tilde{f}$, which always lies below the original $f$ :

Lemma 9 (Dominating $f$ ) Suppose that $n=2$. If we replace the copula $C$ in the proof of Theorem 8 by $C_{L}$ to obtain a new function $\tilde{f}$, then $\tilde{f} \leq f$. 
Proof. Let $\left(V_{1}, V_{2}\right)$ and $\left(V_{1}^{\prime}, V_{2}^{\prime}\right)$ be two pairs of uniform $(0,1)$ random variables with copula $C$ and $C_{L}$ respectively, and $U$ any uniform $(0,1)$ random variable independent of $V_{1}, V_{2}, V_{1}^{\prime}, V_{2}^{\prime}$. For any $\beta \in[0,1]$,

$$
\begin{aligned}
f(\beta)-\tilde{f}(\beta)=\mathbb{E} & {\left[\prod_{i=1}^{2} F_{i}^{-1}\left(I_{\{U>\beta\}} U+I_{\{U \leq \beta\}} \beta V_{i}\right)\right] } \\
& -\mathbb{E}\left[\prod_{i=1}^{2} F_{i}^{-1}\left(I_{\{U>\beta\}} U+I_{\{U \leq \beta\}} \beta V_{i}^{\prime}\right)\right] \\
= & \beta\left\{\mathbb{E}\left[\prod_{i=1}^{2} F_{i}^{-1}\left(\beta V_{i}\right)\right]-\mathbb{E}\left[\prod_{i=1}^{2} F_{i}^{-1}\left(\beta V_{i}^{\prime}\right)\right]\right\},
\end{aligned}
$$

which is positive because $\left(F_{1}^{-1}\left(\beta V_{1}\right), F_{2}^{-1}\left(\beta V_{2}\right)\right)$ and $\left(F_{1}^{-1}\left(\beta V_{1}^{\prime}\right), F_{2}^{-1}\left(\beta V_{2}^{\prime}\right)\right)$ have the same marginal distributions but the latter is counter-comonotonic.

From this lemma we find that if $C_{L}$ is used as the base copula, the corresponding $\tilde{\beta}^{*}$ will be the smallest possible to determine a upper comonotonic random vector $\left(Y_{1}^{\tilde{\beta}^{*}}, Y_{2}^{\tilde{\beta}^{*}}\right)$, in the sense that for all $\left(X_{1}, X_{2}\right) \in \mathcal{R}$ it holds that

$$
X_{1}+X_{2} \leq_{t c x(k)} Y_{1}^{\tilde{\beta}^{*}}+Y_{2}^{\tilde{\beta}^{*}}
$$

with $k$ equals $a_{1}^{\tilde{\beta}^{*}}+a_{2}^{\tilde{\beta}^{*}}$, and there exists no $l<k \in \mathbb{R}$ nor other upper comonotonic vector in $\mathcal{R}_{K}$ for which such relation holds.

\section{Equivalence between convex order and vari- ance order}

Since the variance order is weaker than the convex order in general, it follows that increasing the correlations between the marginal risks $X_{1}, \ldots, X_{n}$, hence increasing the variance of their sum $S$, may not imply that the sum $S$ becomes larger with respect to the convex order, and thus more dangerous to risk averse decision makers. On the other hand, we also know from Theorem 6 that in the extreme case of comonotonicity, we always have such an equivalence between convex order and variance order. The question arises naturally whether there are no situations where increasing variance of $S$ is always equivalent with increasing danger of $S$. In other words, if $\left(X_{1}, \ldots, X_{n}\right)$ and $\left(Y_{1}, \ldots, Y_{n}\right)$ have the same marginals, is it possible for

$$
\sum_{i=1}^{n} X_{i} \leq_{\text {var }} \sum_{i=1}^{n} Y_{i} \Longleftrightarrow \sum_{i=1}^{n} X_{i} \leq_{c x} \sum_{i=1}^{n} Y_{i}
$$

to hold true? In this section, we will demonstrate several instances in which (10) holds true. In these situations, we can compare the riskiness of portfolios with same marginals by simply comparing their variance, which is a much easier task in general. 


\subsection{Location-scale family of distributions}

To begin with, we consider the location-scale family of distributions. We will say that a random variable $X$ belongs to the location-scale family with generator $Z$ if there exist positive real constants $a$ and $b$ such that

$$
X \stackrel{d}{=} a \cdot Z+b .
$$

As the first example, we show that when sums of risks belong to the same location scale family of distributions we find that increasing variance is equivalent with increasing convex ordering.

Proposition 10 (Location-scale family of distributions) If the sums $S_{X}=$ $\sum_{i=1}^{n} X_{i}$ and $S_{Y}=\sum_{i=1}^{n} Y_{i}$ belong to the same location-scale family with a common generator $Z$, then

$$
S_{X} \leq_{v a r} S_{Y} \Longleftrightarrow S_{X} \leq_{c x} S_{Y} .
$$

Proof. To prove " $\Longrightarrow$ ", we can assume that $\mathbb{E}[Z]=0$ and $\operatorname{Var}(Z)=1$ without loss of generality. Since $S_{X}$ and $S_{Y}$ belong to the same location-scale family with $Z$, there exist $b>0$ and $c$ such that $S_{X} \stackrel{d}{=} b Z+c$. Simple calculation reveals that $b=\sqrt{\operatorname{Var}\left(S_{X}\right)}$ and $c=\mathbb{E}\left[S_{X}\right]$, so

$$
S_{X} \stackrel{d}{=} \sqrt{\operatorname{Var}\left(S_{X}\right)} \cdot Z+c .
$$

Similarly, we have

$$
S_{Y} \stackrel{d}{=} \sqrt{\operatorname{Var}\left(S_{Y}\right)} \cdot Z+c
$$

Let us observe that

$$
F_{S_{X}}(x) \leq F_{S_{Y}}(x) \text { for } x \leq c,
$$

whereas

$$
F_{S_{X}}(x)>F_{S_{Y}}(x) \text { for } x>c .
$$

From this it follows by the so-called cut criterion (see e.g. Müller and Stoyan (2002)) that $S_{X} \leq_{i c x} S_{Y}$. Together with the fact that $\mathbb{E}\left[S_{X}\right]=\mathbb{E}\left[S_{Y}\right]$, the result follows.

Under the conditions of Proposition 10, we have either $\sum_{i=1}^{n} X_{i} \leq_{c x} \sum_{i=1}^{n} Y_{i}$ or $\sum_{i=1}^{n} Y_{i} \leq_{c x} \sum_{i=1}^{n} X_{i}$ must hold. An important situation where the condition of the proposition holds (i.e., both $S_{X}$ and $S_{Y}$ belong to the same location-scale family with a common generator $)$ is when $\left(X_{1}, \ldots, X_{n}\right)$ and $\left(Y_{1}, \ldots, Y_{n}\right)$ are multivariate elliptically distributed with the same characteristic generator $\phi$. In particular choosing the characteristic generator equal to $\phi(u)=\exp (-u / 2)$ gives rise to a multivariate normal distribution for both $\left(X_{1}, \ldots, X_{n}\right)$ and $\left(Y_{1}, \ldots, Y_{n}\right)$. For more information on elliptical distributions we refer to the extensive survey of Fang et al. (1990). For a recent application of elliptical distributions in finance and actuarial science; see Valdez et al. (2009) as well as the references herein. 


\subsection{Upper comonotonicity and conditional comonotonic- ity}

Let us assume that there exists an injective mapping $I \ni t \mapsto\left(X_{1}^{t}, \ldots, X_{n}^{t}\right) \in$ $\mathcal{R}\left(F_{1}, \ldots, F_{n}\right)$ such that

$$
\begin{aligned}
t_{1} \leq t_{2} & \Longrightarrow X_{1}^{t_{1}}+\cdots+X_{n}^{t_{1}} \leq_{c x} X_{1}^{t_{2}}+\cdots+X_{n}^{t_{2}} \\
& \Longrightarrow \operatorname{Var}\left(X_{1}^{t_{1}}+\cdots+X_{n}^{t_{1}}\right) \leq \operatorname{Var}\left(X_{1}^{t_{2}}+\cdots+X_{n}^{t_{2}}\right),
\end{aligned}
$$

where $I$ is any subset of $\mathbb{R}$. It is evident in such case the three statements are effectively equivalent, and in particular that the variance order will imply the stronger convex order. In what follows, we demonstrate how one can apply this general principle concretely using the notion of upper comonotonicity and conditional comonotonicity.

\subsubsection{Upper-comonotonicity}

We fix two continuous marginals $F_{1}, F_{2}$ and a 2-copula $C$. Define $Y_{i}^{\beta}$ as in (8) with base copula $C$ :

$$
Y_{i}^{\beta}=F_{i}^{-1}\left(I_{\{U>\beta\}} U+I_{\{U \leq \beta\}} \beta V_{i}\right), \quad i=1,2, \quad \beta \in[0,1] .
$$

From the proof of Theorem $8,\left(Y_{1}^{\beta}, Y_{2}^{\beta}\right)$ is upper-comonotonic. We prove that the equivalence between convex order and variance order can be obtained when varying the parameter $\beta$, provided that the base copula $C$ satisfies an additional property. In what follows, we denote the joint distribution of $\left(Y_{1}^{\beta}, Y_{2}^{\beta}\right)$ as $F_{\beta}$, and the sum $Y_{1}^{\beta}+Y_{2}^{\beta}$ as $S_{\beta}$. The notation $z=x \wedge y$ is used to denote $z=$ $\min (x, y)$. Let $\left(b_{1}, b_{2}\right):=\left(F_{1}^{-1}(\beta), F_{2}^{-1}(\beta)\right)$. Cheung (2009) proved that

(a) if $s_{1}>b_{1}$ and $s_{2}>b_{2}$, then $F_{\beta}\left(s_{1}, s_{2}\right)=F_{1}\left(s_{1}\right) \wedge F_{2}\left(s_{2}\right)$;

(b) otherwise, $F_{\beta}\left(s_{1}, s_{2}\right)=F_{\beta}\left(s_{1} \wedge b_{1}, s_{2} \wedge b_{2}\right)$.

Proposition 11 (Upper tail comonotonic sums when $n=2$ ) If the base copula $C$ fulfills the following property:

$$
\gamma \mapsto \gamma \cdot C\left(\frac{s_{1}}{\gamma}, \frac{s_{2}}{\gamma}\right) \text { is decreasing on }\left[\max \left\{s_{1}, s_{2}\right\}, 1\right] \text { for every } s_{1}, s_{2} \in[0,1] \text {, }
$$

then

$$
\begin{aligned}
0 \leq \alpha \leq \beta \leq 1 & \Longleftrightarrow F_{\beta}\left(s_{1}, s_{2}\right) \leq F_{\alpha}\left(s_{1}, s_{2}\right) \quad \text { for every } s_{1}, s_{2} \\
& \Longleftrightarrow S_{\beta} \leq_{c x} S_{\alpha} \\
& \Longleftrightarrow S_{\beta} \leq_{v a r} S_{\alpha} .
\end{aligned}
$$

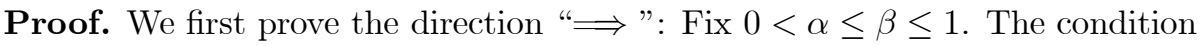
$F_{\beta}\left(s_{1}, s_{2}\right) \leq F_{\alpha}\left(s_{1}, s_{2}\right)$ for every $s_{1}, s_{2}$ is exactly the definition of correlation order: $\left(Y_{1}^{\beta}, Y_{2}^{\beta}\right)$ is smaller than $\left(Y_{1}^{\alpha}, Y_{2}^{\alpha}\right)$ in the correlation order, see for example 
Dhaene and Goovaerts (1996). It was proven there that the correlation ordering implies the convex ordering of the sum and thus the variance ordering as well. Thus, it is enough to prove the right-hand implication in the first line of (13). To this end let $\left(b_{1}, b_{2}\right):=\left(F_{1}^{-1}(\beta), F_{2}^{-1}(\beta)\right)$ and $\left(a_{1}, a_{2}\right):=\left(F_{1}^{-1}(\alpha), F_{2}^{-1}(\alpha)\right)$. Note that $b_{1} \geq a_{1}$ and $b_{2} \geq a_{2}$. When $s_{1} \leq a_{1}$ and $s_{2} \leq a_{2}$,

$$
\begin{aligned}
F_{\alpha}\left(s_{1}, s_{2}\right) & =\alpha \cdot \mathbb{P}\left(\alpha V_{1} \leq F_{1}\left(s_{1}\right), \alpha V_{2} \leq F_{2}\left(s_{2}\right)\right) \\
& =\alpha \cdot C\left(\frac{F_{1}\left(s_{1}\right)}{\alpha}, \frac{F_{2}\left(s_{2}\right)}{\alpha}\right) \geq \beta \cdot C\left(\frac{F_{1}\left(s_{1}\right)}{\beta}, \frac{F_{2}\left(s_{2}\right)}{\beta}\right)=F_{\beta}\left(s_{1}, s_{2}\right),
\end{aligned}
$$

where the inequality follows from assumption (12). When $s_{1}>a_{1}$ and $s_{2}>a_{2}$,

$$
F_{\alpha}\left(s_{1}, s_{2}\right)=F_{1}\left(s_{1}\right) \wedge F_{2}\left(s_{2}\right) \geq F_{\beta}\left(s_{1}, s_{2}\right)
$$

because $F_{1}\left(s_{1}\right) \wedge F_{2}\left(s_{2}\right)$ is the Fréchet upper bound of all joint distribution functions at $\left(s_{1}, s_{2}\right)$ with marginals $F_{1}$ and $F_{2}$. When $s_{1} \leq a_{1}$ and $s_{2}>a_{2}$, and

$$
F_{\alpha}\left(s_{1}, s_{2}\right)=F_{\alpha}\left(s_{1}, a_{2}\right)=\alpha \cdot C\left(\frac{F_{1}\left(s_{1}\right)}{\alpha}, 1\right)=F_{1}\left(s_{1}\right)
$$

and

$$
F_{\beta}\left(s_{1}, s_{2}\right)=F_{\beta}\left(s_{1}, s_{2} \wedge b_{2}\right)=\beta \cdot C\left(\frac{F_{1}\left(s_{1}\right)}{\beta}, \frac{F_{2}\left(s_{2} \wedge b_{2}\right)}{\beta}\right),
$$

thus we have

$$
\begin{aligned}
F_{\alpha}\left(s_{1}, s_{2}\right)=F_{1}\left(s_{1}\right) & =\beta \cdot C\left(\frac{F_{1}\left(s_{1}\right)}{\beta}, 1\right) \\
& \geq \beta \cdot C\left(\frac{F_{1}\left(s_{1}\right)}{\beta}, \frac{F_{2}\left(s_{2} \wedge b_{2}\right)}{\beta}\right)=F_{\beta}\left(s_{1}, s_{2}\right) .
\end{aligned}
$$

Similarly, one can prove that $F_{\alpha}\left(s_{1}, s_{2}\right) \geq F_{\beta}\left(s_{1}, s_{2}\right)$ when $s_{1}>a_{1}$ and $s_{2} \leq a_{2}$. Combining all these cases together yields $F_{\alpha}\left(s_{1}, s_{2}\right) \geq F_{\beta}\left(s_{1}, s_{2}\right)$ for all $s_{1}$ and $s_{2}$. For the proof of "£": Suppose that $S_{\beta} \leq_{v a r} S_{\alpha}$. If $\alpha>\beta$, the proof

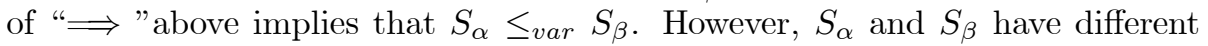
distributions by construction. This leads to a contradiction and hence we must have $\alpha \leq \beta$.

We remark that condition (12) is fulfilled by some commonly used copula, like the independence copula $C_{I}(u, v)=u v$ and the Fréchet lower bound copula $C_{L}(u, v)=\max \{u+v-1,0\}$. The underlying meaning of Proposition 11 is that when the parameter $\beta$ gets smaller, the random vector $\left(Y_{1}^{\beta}, Y_{2}^{\beta}\right)$ is comonotonic over a larger upper quadrant, and hence the sum $S_{\beta}$ becomes convex bigger. We also remark that if the dimension $n$ is strictly greater than two, the construction above no longer works. While $F_{\beta} \leq F_{\alpha}$ still holds true when $\alpha \leq \beta$ when the base copula fulfills the $n$-dimensional analog of condition (12), it is known that such an ordering of the joint distribution functions is not strong enough to guarantee a corresponding convex order of the sums. 


\subsubsection{Conditional comonotonicity}

To apply the general principle stated at the beginning Section 5.2 in higher dimensions, we consider a notion called conditional comonotonicity. Loosely speaking a random vector is comonotonic conditional on a certain sub- $\sigma$-field if the corresponding regular conditional distribution is comonotonic almost surely, see Jouini and Napp (2004) for the precise definition and the rationale behind the concept. Cheung (2007) proved, under some topological assumptions on the underlying sample space, that conditional comonotonicity is equivalent to local comonotonicity on each atom of the sub- $\sigma$-field. A proof for the following result can be found in Cheung (2008a).

Proposition 12 (conditionally comonotonic sums) Given the marginal distributions $F_{1}, \ldots, F_{n}$ and filtration $\mathcal{F}_{1} \subseteq \mathcal{F}_{2} \subseteq \cdots$, there exists a sequence of random vectors $\mathbf{Y}^{1}, \mathbf{Y}^{2}, \ldots$ in $\mathcal{R}\left(F_{1}, \ldots, F_{n}\right)$ so that

$$
S^{1} \geq_{c x} S^{2} \geq_{c x} \geq S^{3} \geq_{c x} \cdots,
$$

where $S^{k}:=Y_{1}^{k}+\cdots+Y_{n}^{k}$, and each $\mathbf{Y}^{k}$ is comonotonic conditional on $\mathcal{F}_{k}$.

Because of (14), the family $\left\{\mathbf{Y}^{1}, \mathbf{Y}^{2}, \ldots\right\}$ provides an $n$-dimensional example of the equivalence between the convex order and the variance order of the sums:

$$
S^{m} \leq_{c x} S^{n} \Longleftrightarrow S^{m} \leq_{\text {var }} S^{n}, \quad m \leq n .
$$

The idea here is similar to that of Proposition 11. When the index $k$ is larger, the atoms in $\mathcal{F}_{k}$ becomes smaller and so $\mathbf{Y}^{k}$ is comonotonic over a larger number of bigger regions. This makes the sum $S^{k}$ convex smaller.

\section{Acknowledgments}

The authors wish to thank the anonymous referee for several valuable comments and suggestions which significantly improved the manuscript. K.C. Cheung's work was supported by Research Grants Council of HKSAR (Project No.: HKU701409P) and the Seed Funding Programme for Basic Research of The University of Hong Kong (Project No.:200905159011). Steven Vanduffel acknowledges the financial support of the BNP Paribas Fortis Chair in Banking.

\section{References}

[1] Campana, A. (2007). Tail Value-at-Risk for sums of non-independent random variables with a generalized Pareto distribution, GENEVA Risk and Insurance Review, 32, 169-180.

[2] Cheung, K.C. (2007). Characterizations of conditional comonotonicity. Journal of Applied Probability, 44, 607-617. 
[3] Cheung, K.C. (2008a). Improved convex upper bound via conditional comonotonicity. Insurance: Mathematics and Economics, 42, 651-655.

[4] Cheung, K.C. (2008b). Characterization of comonotonicity using convex order. Insurance: Mathematics and Economics, 43, 403-406.

[5] Cheung, K.C. (2009). Upper comonotonicity. Insurance: Mathematics and Economics, 45, 35-40.

[6] Cuesta, J.A., Matran, C. (1989). Notes on the Wasserstein metric in Hilbert spaces. Ann. Probab., 17, 1264-1276.

[7] Deelstra, G., Dhaene, J., Vanmaele, M., 2010. An overview of comonotonicity and its applications in finance and insurance. Advanced Mathematical Methods for Finance (Editors: Øksendal, B., Nunno, G.), Springer, Germany.

[8] Deelstra, G., Diallo, I., Vanmaele, M. (2008). Bounds for Asian basket options, Journal of Computational and Applied Mathematics, 218, 215228.

[9] Denneberg, D. (1994). Non-additive measure and integral. Kluwer Academic Publishers, Boston.

[10] Denuit, M., Dhaene, J., Van Wouwe, M. (1999). The economics of insurance: a review and some recent developments. Mitteilungen der Schweiz. Aktuarvereinigung. 2, 137-175.

[11] Denuit, M., Dhaene, J., Goovaerts, M.J., Kaas, R. (2005). Actuarial Theory for Dependent Risks: Measures, Orders and Models, John Wiley \& Sons, Inc.

[12] Dhaene, J., Denuit, M., Goovaerts, M.J., Kaas, R., Vyncke, D., (2002). The concept of comonotonicity in actuarial science: theory. Insurance: Mathematics and Economics, 31, 3-33.

[13] Dhaene, J., Goovaerts, M. (1996). Dependence of risks and stop-loss order. ASTIN Bulletin, 26, 201-212.

[14] Dhaene, J., Goovaerts, M., Vanduffel, S. (2008). Comonotonicity. Encyclopedia of Quantitative Risk Assessment and Analysis, Melnick, E. and Everitt, B. (eds). John Wiley \& Sons Ltd, Chichester, UK, 274-279.

[15] Dhaene, J., Vanduffel, S., Tang, Q., Goovaerts, M.J., Kaas, R., Vyncke, D. (2006). Risk measures and comonotonicity: a review. Stochastic Models, $22,573-606$.

[16] Dong, J., Cheung, K.C., Yang, H. (2010). Upper comonotonicity and convex upper bounds for sums of random variables. To appear in Insurance Mathematics and Economics. 
[17] Embrechts, P., Pucetti, G. (2006). Aggregating risk capital, with an application to operational risk, GENEVA Risk and Insurance Review, 31, 71-90.

[18] Fang, K.T., Kotz, S., Ng, K.W. (1990). Symmetric Multivariate and Related Distributions. London: Chapman \& Hall.

[19] Föllmer, H., Schied, A., (2004). Stochastic Finance. An introduction in discrete time, Second edition, Walter de Gruyter, Berlin

[20] Jouini, E., Napp, C. (2004). Conditional comonotonicity. Decisions In Economics and Finance, 27, 153-166.

[21] Kaas, R., Dhaene, J., Vyncke, D., Goovaerts, M. J., Denuit, M. (2002). A simple geometric proof that comonotonic risks have the convex-largest sum. ASTIN Bull., 32, 71-80.

[22] Landsman, Z., Tsanakas, A. (2006). Stochastic ordering of bivariate elliptical distributions. Statistics and Probability Letters, 76, 488-494.

[23] Müller, A., Stoyan, D. (2002). Comparison Methods for Stochastic Models and Risks. John Wiley \& Sons, Inc.

[24] Valdez, E., Dhaene, J., Maj, M., Vanduffel, S. (2009). Bounds and approximations for sums of dependent log-elliptical random variables. Insurance: Mathematics and Economics, 44, 385-397.

[25] Vandendorpe, A., Ho, N., Vanduffel, S. Van Dooren, P. (2008). On the parameterisation of the CreditRisk+ model for estimating credit portfolio risk. Insurance: Mathematics and Economics, 42, 736-745.

[26] Vanduffel, S., Shang, Z., Shang, Z., Henrard, L., Dhaene, J., Valdez, E. (2008a). Analytic bounds and approximations for Annuities and Asian options. Insurance: Mathematics and Economics, 42(3), 1109-1117.

[27] Vanduffel, Chen, X., Dhaene, J., Goovaerts, M., Henrard, L. (2008b). Optimal approximations for risk measures of sums of lognormals based on conditional expectations. Journal of Computational and Applied Mathematics, 221(1), 202-218.

[28] Vanmaele, M., Deelstra, G., Liinev, J. (2004) Approximation of stop-loss premiums involving sums of lognormals by conditioning on two variables, Insurance Mathematics and Economics, 35, 343-368. 